\title{
A Second Law Based Unstructured Finite Volume Procedure for Generalized Flow Simulation
}

\author{
Alok Majumdar \\ Sverdrup Technology \\ Huntsville, Alabama
}

\begin{abstract}
An unstructured finite volume procedure has been developed for steady and transient thermo-fluid dynamic analysis of fluid systems and components. The procedure is applicable for a flow network consisting of pipes and various fittings where flow is assumed to be one dimensional. It can also be used to simulate flow in a component by modeling a multi-dimensional flow using the same numerical scheme. The flow domain is discretized into a number of interconnected control volumes located arbitrarily in space. The conservation equations for each control volume account for the transport of mass, momentum and entropy from the neighboring control volumes. In addition, they also include the sources of each conserved variable and time dependant terms. The source term of entropy equation contains entropy generation due to heat transfer and fluid friction. Thermodynamic properties are computed from the equation of state of a real fluid. The system of equations is solved by a hybrid numerical method which is a combination of simultaneous Newton-Raphson and successive substitution schemes. The paper also describes the application and verification of the procedure by comparing its predictions with the analytical and numerical solution of several benchmark problems.
\end{abstract}

\section{NOMENCLATURE}

$\begin{array}{cl}\text { A } & \left.\text { Area (in }{ }^{2}\right) \\ \mathrm{A}_{0} & \text { Pump Characteristic Curve Coefficient } \\ \mathrm{B}_{0} & \text { Pump Characteristic Curve Coefficient } \\ \mathrm{D} & \text { Diameter (in) } \\ \mathrm{f} & \text { Darcy Friction Factor } \\ \mathrm{g} & \text { Gravitational Acceleration }\left(\mathrm{ft} / \mathrm{sec}^{2}\right) \\ \mathrm{g}_{\mathrm{c}} & \text { Conversion Constant }\left(=32.174 \mathrm{lb}-\mathrm{ft} / \mathrm{lb}_{\mathrm{r}}-\mathrm{sec}^{2}\right) \\ \mathrm{J} & \text { Mechanical Equivalent of Heat }\left(778 \mathrm{ft}-\mathrm{lb}_{\mathrm{f}} / \mathrm{Btu}\right) \\ \mathrm{K}_{\mathrm{f}} & \text { Flow Resistance Coefficient }\left(/ \mathrm{lb} \mathrm{b}_{\mathrm{f}} \mathrm{sec}^{2} /(\mathrm{lb}-\mathrm{ft})^{2}\right) \\ \mathrm{K}_{\mathrm{rot}} & \text { Non-dimensional Rotating Flow Resistance Coefficient } \\ \mathrm{L} & \text { Length (in) } \\ \mathrm{M} & \text { Molecular Weight } \\ \mathrm{m} & \text { Resident Mass }(\mathrm{lb}) \\ \dot{m} & \text { Mass Flow Rate }(\mathrm{lb} / \mathrm{sec}) \\ \mathrm{p} & \text { Pressure }\left(\mathrm{lb} / \mathrm{in}{ }^{2}\right) \\ \mathrm{Q} & \text { Heat Source }(\mathrm{Btu} / \mathrm{sec})\end{array}$




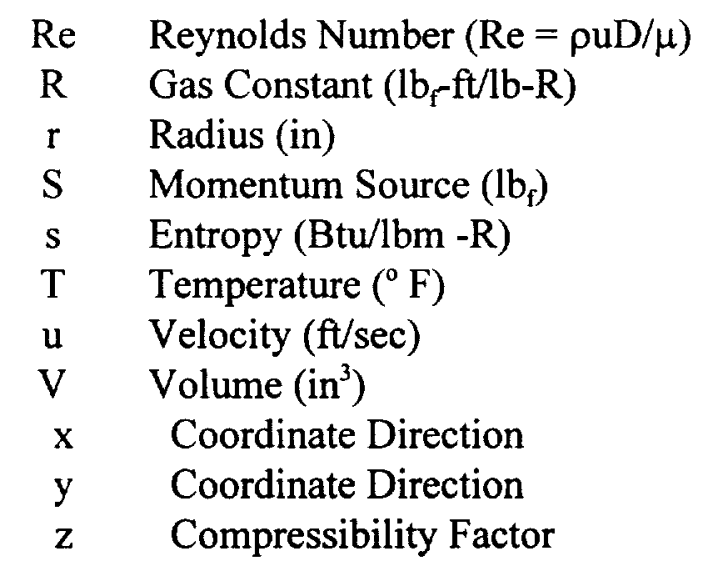

\section{Greek}

$\begin{array}{cl}\rho & \text { Density }\left(\mathrm{lb} / \mathrm{ft}^{3}\right) \\ \theta & \text { Angle Between Branch Flow Velocity Vector and Gravity Vector (deg), } \\ & \text { Angle Between Neighboring Branches for Computing Shear (deg) } \\ \omega & \text { Angular Velocity (rad/sec) } \\ \varepsilon & \text { Absolute Roughness (in) } \\ \varepsilon / D & \text { Relative Roughness } \\ \mu & \text { Viscosity ( lb/ft-sec) } \\ \gamma & \text { Specific Heat Ratio } \\ \Delta \tau & \text { Time Step (sec) } \\ \tau & \text { Time (sec) }\end{array}$

\section{INTRODUCTION}

A numerical procedure capable of modeling fluid flow for both systems and components is of significant importance in aerospace and many other engineering industries. Although separate numerical methods are available for performing system and component analysis, there are not many attempts to develop an unified procedure applicable to both systems and components. Hardy Cros $\mathrm{s}^{1}$ developed a numerical method for calculating flow and pressure distribution in a flow network. This method has been widely used in modeling water distribution systems in municipalities. The Hardy Cross method assumes an equilibrium between pressure and friction forces in steady and incompressible flow. As a result it has not been successfully used in unsteady and compressible flow calculations where inertia force is important.

Patankar and Spalding ${ }^{2}$ developed a finite volume procedure to solve for Navier-Stokes equations in a structured co-ordinate system. Since the publication of the original paper in 1972, there have been several developments reported to improve the numerical performance of the original algorithm. Several general purpose computer programs that have been developed based on these procedures, are increasingly being used for 
component analysis. However, very few applications of the finite volume method have been reported for system analysis. Datta and Majumdar ${ }^{3}$ used this solution algorithm to develop a calculation procedure for manifold flow systems. However, this procedure can not be used for simulating a flow network due to its dependence on a structured coordinate system. The purpose of this paper is to present a finite volume procedure based on an unstructured co-ordinate system that can be applied to analyze both systems and components.

\section{MATHEMATICAL FORMULATION}

All finite volume procedures require solution of mass, momentum and energy conservation equations in a flow domain consisting of interconnected control volumes. For turbulent flows, additional conservation equations of turbulence parameters are solved to calculate enhanced momentum exchanges due to turbulence. For reactive flows, specie conservation equations are solved in conjunction with other conservation equations. The main characteristic features of the present method are the following:

\section{Unstructured Co-ordinate System}

A flow domain is resolved into a network consisting of nodes and branches. The nodes are connected by branches. The nodes are classified into two categories: boundary and internal nodes. At a boundary node, pressure and temperature are known; at an internal node they are computed by solving conservation equations. Each internal node can be connected with an arbitrary number of internal and boundary nodes. At each node mass and entropy conservation equations are solved in conjunction with the equation of state. The momentum conservation equations are solved at all branches. This process of discretization allows the development of the set of conservation equations in an unstructured co-ordinate system. A schematic of a typical flow network is shown in Figure 1.

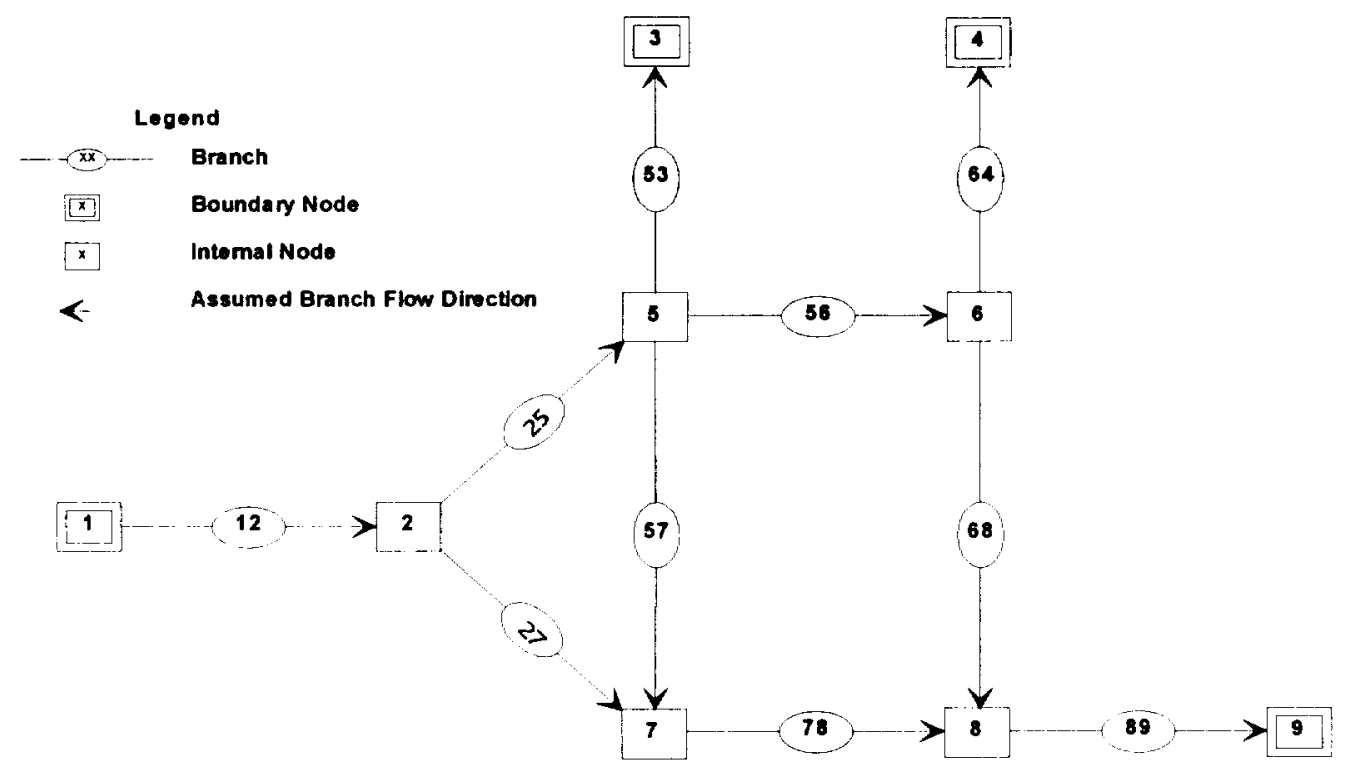

Figure 1 - Schematic of a Flow System Consisting of Nodes and Branches 3

Draft paper for $37^{\text {th }}$ AIAA Aerospace Sciences Meeting 


\section{Viscous Effect}

In conventional finite volume procedure, viscous effects are modeled by shear stress, which is expressed as a product of effective viscosity and local velocity gradient. In order to calculate the velocity gradient accurately, the finite volume procedure requires a large number of control volumes. It is not practical to use such a large mesh for a system level calculation. Therefore, in this method the frictional effect is modeled in the momentum equation by the following expression:

$$
\Delta p_{\text {friction }}=K_{f} \dot{m}^{2}
$$

where $K_{f}$ is a function of Reynolds number, density, area and geometry of the branch in consideration. However for multi-dimensional flows, viscous effects are modeled by conventional shear stress method.

\section{Thermodynamic Formulation}

Entropy is calculated at every node using a second law analysis ${ }^{4}$ of the control volume. The temperature and density at an internal node are calculated from pressure and entropy using a modified virial equation of state ${ }^{5}$. During each iteration cycle, thermodynamic properties are calculated to ensure thermodynamic equilibrium prevails during the course of attaining the solution.

\section{Solution Scheme}

The system of equations is solved by a hybrid numerical scheme which is a combination of the Newton-Raphson and successive substitution schemes. The equations which are strongly coupled are solved by the Newton-Raphson method. The equations which are weakly coupled are solved by the successive substitution scheme. The successive substitution scheme is also used to develop an initial guess for the Newton-Raphson scheme to ensure numerical stability.

\section{GOVERNING EQUATIONS}

Figure 2 displays a schematic showing adjacent nodes, their connecting branches, and the indexing system used in this paper. In order to solve for the unknown variables, mass and entropy conservation equations and the equation of thermodynamic state are written for each internal node and flow rate equations are written for each branch.

\section{Mass Conservation Equation}

$$
\frac{m_{\tau+\Delta \tau}-m_{\tau}}{\Delta \tau}=\sum_{j=1}^{j=n} m_{i j}
$$




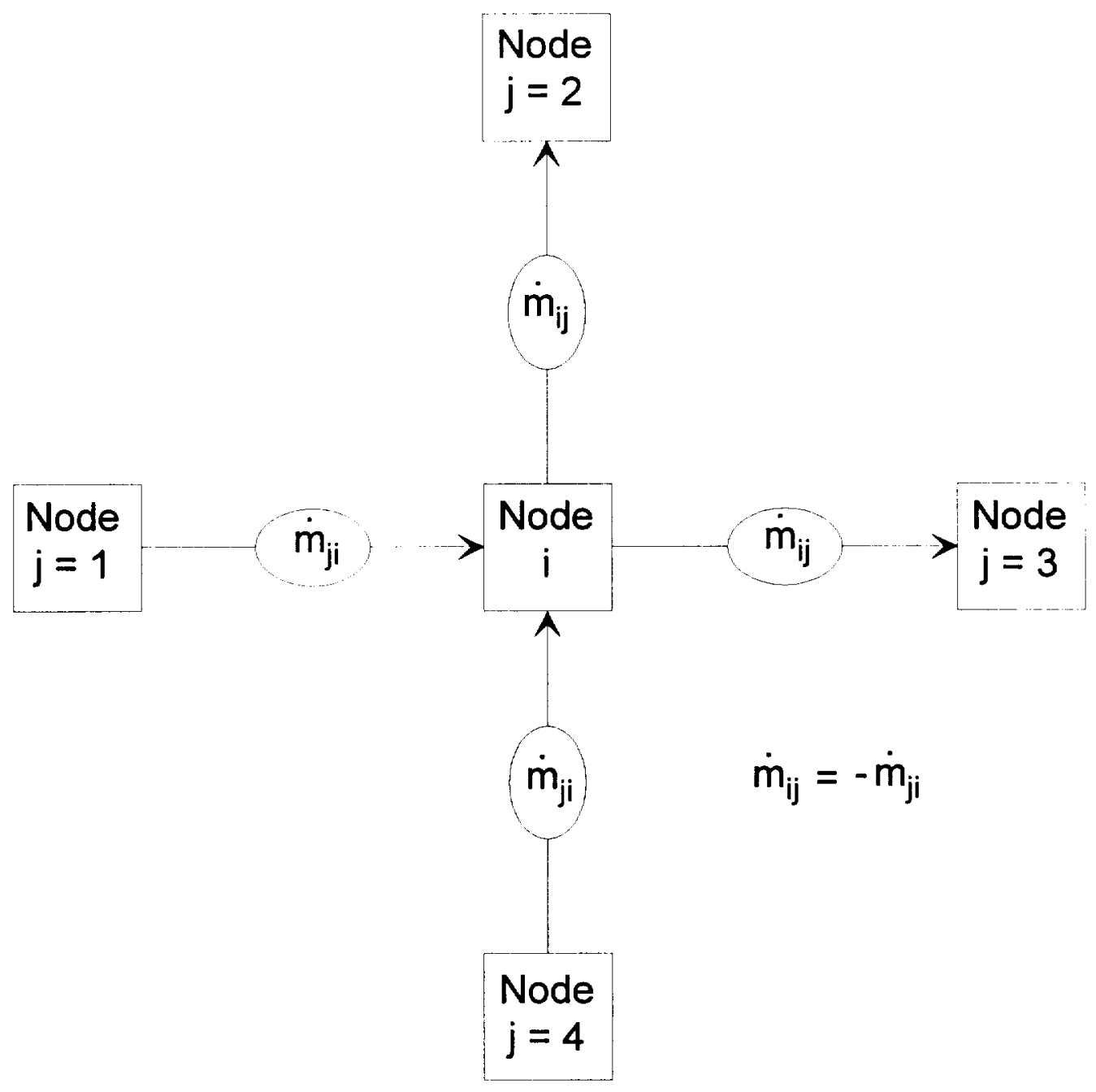

Figure 2 - Schematic of Nodes, Branches and Indexing Practice

Equation 2 requires that, for the transient formulation, the net mass flow from a given node must equate to the rate of change of mass in the control volume. In the steady state formulation, the left hand side of the equation is zero. This implies that the total mass flow rate into a node is equal to the total mass flow rate out of the node.

\section{Momentum Conservation Equation}

The flow rate in a branch is calculated from the momentum conservation equation (Equation 3) which represents the balance of fluid forces acting on a given branch. A typical branch configuration is shown in Figure 3. Inertia, pressure, gravity, friction and centrifugal forces are considered in the conservation equation. In addition to these five forces, a source term $\mathrm{S}$ has been provided to represent any external momentum sources. For example, this external momentum source term can be used to model a pump in a flow system. 


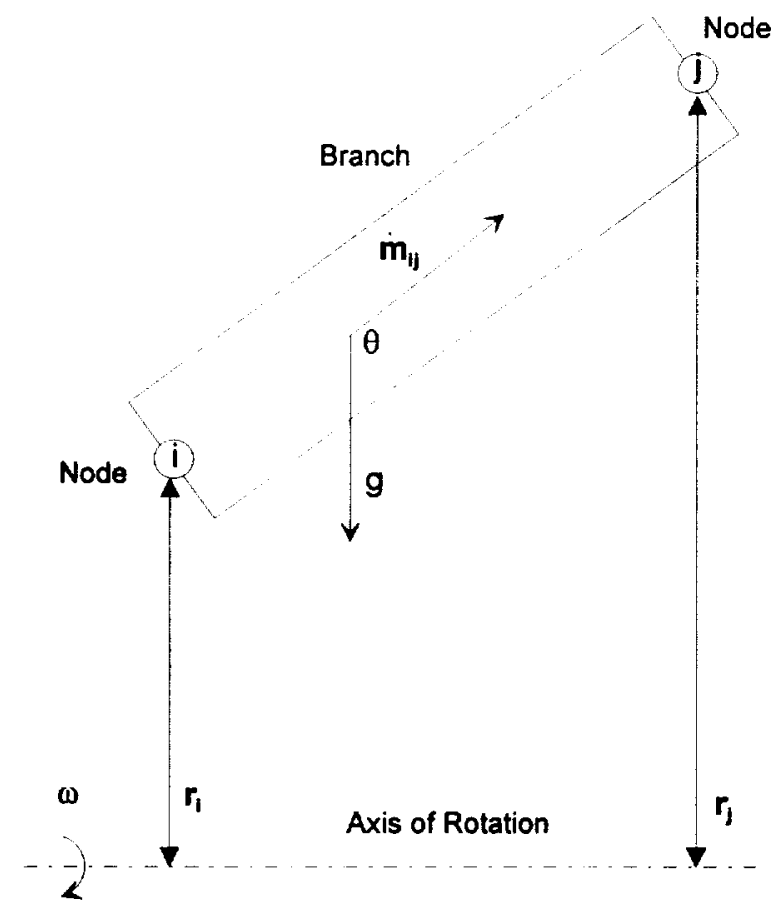

Figure 3 - Schematic of a Branch Showing Gravity and Rotation

$$
\begin{aligned}
& \frac{\left(m u_{\tau+\Delta \tau}-m u_{\tau}\right)}{g_{c} \Delta \tau}+\frac{\dot{m_{i j}}}{g_{c}}\left(u_{i j}-u_{u}\right)= \\
& \left(p_{i}-p_{j}\right) A+\frac{\rho g V \cos \theta}{g_{c}}-K_{f} \dot{m}_{i j}\left|\dot{m}_{i j}\right| A+\frac{\rho K_{r o t}^{2} \alpha^{2} A}{2 g_{c}}\left(r_{j}^{2}-r_{i}^{2}\right)+S
\end{aligned}
$$

The two terms in the left hand side of the momentum equation represent the inertia of the fluid. The first one is the time dependent term and must be considered for unsteady calculations. The second term is significant when there is a large change in area or density from branch to branch. The first term in the right hand side of the momentum equation represents the pressure gradient in the branch. The pressures are located at the upstream and downstream face of a branch. The second term represents the effect of gravity. The gravity vector makes an angle $(\theta)$ with the assumed flow direction vector. The third term represents the frictional effect. Friction was modeled as a product of $K_{f}$ and the square of the flow rate and area. $\mathrm{K}_{\mathrm{f}}$ is a function of the fluid density in the branch and the nature of the flow passage being modeled by the branch. The calculation of $K_{f}$ for different types of flow passages is described later in this paper. The fourth term in the momentum equation represents the effect of the centrifugal force. This term will be present only when the branch is rotating as shown in Figure $3 . K_{\text {rot }}$ is the factor representing the fluid rotation. $\mathrm{K}_{\mathrm{rot}}$ is unity when the fluid and the surrounding solid 
surface rotates with the same speed. This term also requires a knowledge of the distances between the upstream and downstream faces of the branch from the axis of rotation.

\section{Entropy Conservation Equation}

The entropy conservation equation for node i, shown in Figure 2, can be expressed mathematically as shown in Equation 4.

$$
\begin{aligned}
& \frac{(m s)_{\tau+\Delta \tau}-(m s)_{\tau}}{\Delta \tau}= \\
& \sum_{j=1}^{j=n}\left\{M A X\left[-\dot{m}_{i j}, 0\right] s_{j}-M A X\left[\dot{m}_{i j}, 0\right] s_{i}\right\}+\sum_{j=1}^{j=n}\left\{\frac{M A X\left[m_{j i}, 0\right]}{\left|m_{j j}\right|}+\frac{M A X\left[-\dot{m}_{i j}, 0\right]}{\left|\dot{m}_{i j}\right|}\right\} \mathrm{S}_{\mathrm{ij}, \mathrm{gen}}+\frac{Q_{i}}{T_{i}}
\end{aligned}
$$

The entropy generation rate due to fluid friction in a branch is expressed as

$$
\dot{S}_{i j, j e n}=\frac{\dot{m}_{i j} \Delta p_{i j, v i s c o u s}}{\rho_{u} T_{u} J}=\frac{K_{f}\left(\left|\dot{m}_{i j}\right|\right)^{3}}{\rho_{u} T_{u} J}
$$

Equation 4 shows that for unsteady flow, the rate of increase of entropy in the control volume is equal to the rate of entropy transport into the control volume plus the rate of entropy generation in all upstream branches due to fluid friction plus the rate of entropy added to the control volume due to heat transfer. The MAX operator used in Equation 4 is known as an upwind differencing scheme ${ }^{2}$ which has been extensively employed in the numerical solution of Navier-Stokes equations in convection heat transfer and fluid flow applications. When the flow direction is not known, this operator allows the transport of entropy only from its upstream neighbor. In other words, the upstream neighbor influences its downstream neighbor but not vice versa.

Equation of State

A modified virial equation of state ${ }^{5}$ is used to calculate the density from the computed pressure and temperature at a given node.

$p=\sum_{i=1}^{i=N} A_{i}(T) \rho^{i}+\sum_{j=1}^{j=M} B_{j}(T) \rho^{2 j+1} e^{-c \rho^{2} / T_{2}}$

$A_{i}(T)$ and $B_{j}(T)$ are polynomials in $T$ and $1 / T$

This equation was originally developed by Benedict, Webb and Rubin (BWR) and later modified by Bender ${ }^{5}$. This equation was the basis of the computer code GASP developed 
by Hendricks et al ${ }^{6}$. This equation enabled PVT calculations to be made in the liquid and vapor phases. The derived properties of enthalpy and entropy were also obtained. The present method uses the GASP computer code to compute density and other thermophysical properties during iterative cycles of computation.

\section{Compressibility Factor}

For unsteady flow, resident mass in a control volume is calculated from the compressibility factor assuming a thermodynamic equilibrium.

$$
m=\frac{p V}{R T z}
$$

The following table shows how each equation is used to calculate the unknown variables to demonstrate the problem of mathematical closure.

Table 1 - Mathematical Closure

\begin{tabular}{|c|c|}
\hline $\begin{array}{c}\text { Unknown } \\
\text { variables }\end{array}$ & Available equations to solve \\
\hline Pressure & Mass Conservation Equation (Eqn. 1) \\
\hline Flow rate & Momentum Conservation Equation (Eqn. 2) \\
\hline Temperature & Second Law of Thermodynamics (Eqn. 3) \\
\hline Density & Equation of State (Eqn. 4) \\
\hline $\begin{array}{c}\text { Mass (Unsteady } \\
\text { Flow only) }\end{array}$ & Compressibility Factor (Eqn 5) \\
\hline
\end{tabular}

$\underline{\text { Multi-Dimensional Flow }}$

Multi-dimensional conservation equations must account for the transport of mass, momentum and entropy into and out of the control volume from all directions in space. The scalar transport equations (i. e., mass and entropy conservation equations) can account for such transport because each internal node can be connected with multiple neighboring nodes located in space in any arbitrary locktion (Figure 2). Equations 2 and 4 account for the transport of mass and entropy respectively from all directions in space. On the other hand, the momentum conservation equation (Equation 3) is one dimensional. Multi-dimensional momentum transport can be accounted for by incorporating two additional terms in the momentum equation. These terms include: a) momentum transport due to shear, and b) momentum transport due to the transverse component of velocity.

These two terms can be identified in the two-dimensional steady state Navier-Stokes equation which can be expressed as: 


$$
\rho\left(u \frac{\partial u}{\partial x}+v \frac{\partial u}{\partial y}\right)=\frac{\partial(\rho u u)}{\partial x}+\frac{\partial(\rho v u)}{\partial y}=-\frac{\partial p}{\partial x}+\rho g_{x}+\mu\left(\frac{\partial^{2} u}{\partial x^{2}}+\frac{\partial^{2} u}{\partial y^{2}}\right)
$$

The first term on the left hand side of Equation 7 corresponds to the inertia term in Equation 3. The second term on the left hand side of Equation 7 corresponds to the transverse momentum exchange. The first term on the right hand side of Equation 7 corresponds to the pressure term. The second term on the right hand side of Equation 7 corresponds to the gravity term. The third term on the right hand side of the equation is negligible (based on an order of magnitude argument). The fourth term on the right hand side corresponds to the friction term in the one dimensional momentum conservation equation. For multi-dimensional flow friction is modeled by shear force as discussed below.

Momentum Transport Due to Shear

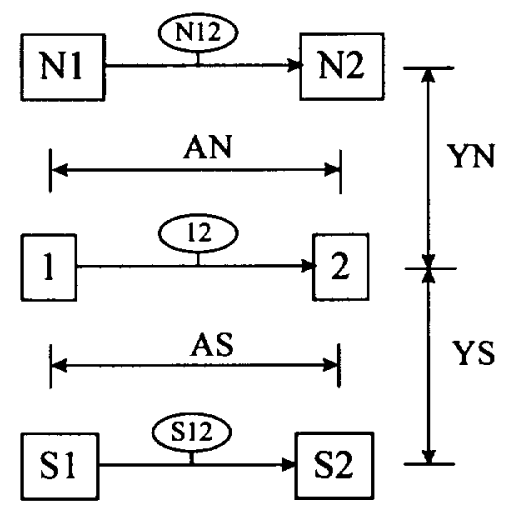

Figure 4 - Branch and Node Schematic for Shear Exchange

Figure 4 represents a set of nodes and branches for which shear forces are exchanged. Let branch 12 represent the branch for which the shear force is to be calculated. Branches $\mathrm{N} 12$ and S12 represent the parallel branches which will be used to calculate the shear force on branch 12. Let YS be the distance between branches 12 and $\mathrm{S} 12$, and let $\mathrm{YN}$ be the distance between branches 12 and N12. Let AS be the shearing area between branches 12 and S12, while AN is the shearing area between branches 12 and N12.

The shear force on a control volume can be expressed as

$\mu \frac{\partial^{2} \mathrm{u}}{\partial \mathrm{y}^{2}} \mathrm{~V} \approx \mu \frac{\Delta \mathrm{u}}{(\Delta \mathrm{y})^{2}}(\Delta \mathrm{x})(\Delta \mathrm{y})(\Delta \mathrm{z})=\mu \frac{\Delta \mathrm{u}}{\Delta \mathrm{y}} \Delta \mathrm{x} \Delta \mathrm{z}=\mu \frac{\Delta \mathrm{u}}{\Delta \mathrm{y}} \mathrm{A}_{\text {shear }}$

The finite volume formulation for shear for the $\mathrm{i}^{\text {th }}$ branch can be written as: 
$\mu \frac{\partial \mathrm{u}}{\partial \mathrm{y}} \mathrm{A}_{\text {Branch i shear }} \approx \sum_{\mathrm{j}=1}^{\mathrm{np}}\left(\mu \frac{\mathrm{u}_{\mathrm{ij}} \cos \theta_{\mathrm{ij}}-\mathrm{u}_{\mathrm{i}}}{\mathrm{Y}_{\mathrm{ij}}} \mathrm{A}_{\mathrm{ij}}\right)+\sum_{\mathrm{k}=1}^{\mathrm{ns}}\left(\mu \frac{\mathrm{u}_{\text {wall ik }} \cos \theta_{\text {wall ik }}-\mathrm{u}_{\mathrm{i}}}{\mathrm{y}_{\text {wall ik }}} \mathrm{A}_{\mathrm{ik}}\right)$

where $i$ is the current branch, $n p_{i}$ is the number of parallel branches to branch $i$, and $n s_{i}$ is the number of parallel solid walls to branch $i$.

Transverse Momentum Transport

The transverse momentum component of Equation 7 can be expressed in terms of a force per unit volume.

$$
\frac{\partial \rho v u}{\partial y} \mathrm{~V} \approx \frac{(\rho v \Delta \mathrm{u})}{(\Delta \mathrm{y})}(\Delta \mathrm{x})(\Delta \mathrm{y})(\Delta \mathrm{z})=(\Delta \mathrm{x})(\Delta \mathrm{z})(\rho \mathrm{v} \Delta \mathrm{u})=\dot{\mathrm{m}}_{\mathrm{trans}} \Delta \mathrm{u}
$$

Figure 5 represents a set of nodes and branches for which transverse momentum exchange will take place. Let branch 12 represent the current branch which will receive transverse momentum from the surrounding branches. Branch $\mathrm{S} 12$ represents an adjacent parallel branch, while branches $S 1$ and $S 2$ represent the adjacent normal branches.

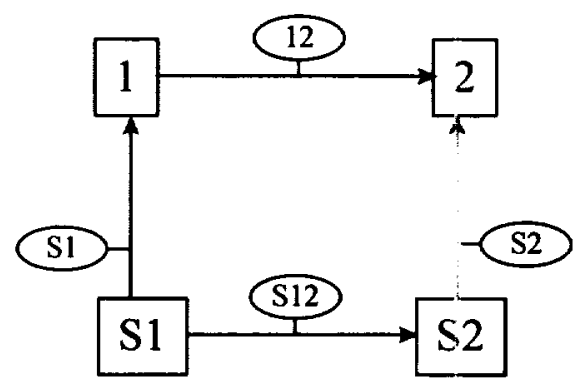

Figure 5 - Branch and Node Schematic for Transverse Momentum Exchange The finite volume formulation for transverse momentum for the $\mathrm{i}^{\text {th }}$ branch can be written as

$$
\left.\frac{\partial \rho v u}{\partial y} v\right|_{i} \approx \sum_{j=1}^{m_{i}}\left[u _ { i } \left(\max \left\|-\sum_{k=1}^{n_{i j}}\left\{\frac{1}{n_{i j}} \dot{m}_{i j k} \cos \theta_{i j k}\right\}, 0\right\|-\left(u_{i j} \cos \theta_{i j}\right)\left\{\max \left\|0, \sum_{k=1}^{n_{i j}}\left\{\frac{1}{n_{i j}} \dot{m}_{i j k} \cos \theta_{j i k}\right\}\right\|\right]\right.\right.
$$

Detailed derivation of equation (9) and (11) appear in GFSSP User's manual ${ }^{7}$.

\section{SOLUTION PROCEDURE}

In the sample circuit shown in Figure 1, pressures and temperatures are to be calculated for the 5 internal nodes; flow rates are to be calculated in the 10 branches. The total 
number of equations can be evaluated from the following relationship: Number of equations $=$ Number of internal nodes $*$ Number of scalar transport equations + Number of branches. Therefore, the total number of equations to be solved is $20(=5 \times 2+10)$.

There is no explicit equation for pressure. The pressures are implicitly computed from the mass conservation equation (Equation 2). The flow rates are calculated from Equation 3. The inertia and friction terms are nonlinear in Equation 3. The pressures and mass flow rates appear in the flow rate equations. The flow rates also appear in the entropy equation. The governing equations to be solved are strongly coupled and nonlinear and therefore they must be solved by an iterative method.

There are two types of numerical methods available to solve a set of non-linear coupled algebraic equations: (1) the successive substitution method and (2) the Newton-Raphson method. In the successive substitution method, each equation is expressed explicitly to calculate one variable. The previously calculated variable is then substituted into the other equations to calculate another variable. In one iterative cycle each equation is visited. The iterative cycle is continued until the difference in the values of the variables in successive iterations becomes negligible. The advantages of the successive substitution method are its simplicity to program and its low code overhead. The main limitation, however, is finding an optimum order for visiting each equation in the model. This visiting order, which is called the information flow diagram, is crucial for convergence. Under-relaxation (partial substitution) of variables is often required to obtain numerical stability.

In the Newton-Raphson method, the simultaneous solution of a set of non-linear equations is achieved through an iterative guess and correction procedure. Instead of solving for the variables directly, correction equations are constructed for all of the variables. The intent of the correction equations is to eliminate the error in each equation. The correction equations are constructed in two steps: (1) the residual errors in all of the equations are estimated and (2) the partial derivatives of all of the equations, with respect to each variable, are calculated. The correction equations are then solved by the Gaussian elimination method. These corrections are then applied to each variable, which completes one iteration cycle. These iterative cycles of calculations are repeated until the residual error in all of the equations is reduced to a specified limit. The Newton-Raphson method does not require an information flow diagram. Therefore, it has improved convergence characteristics. The main limitation to the Newton-Raphson method is its requirement for a large amount of computer memory.

The present formulation employs a novel numerical scheme, SASS (Simultaneous Adjustment with Successive Substitution) which is a combination of the successive substitution and Newton-Raphson methods. The mass and momentum conservation equations are solved by the Newton-Raphson method. The entropy conservation equations are solved by the successive substitution method. The underlying principle for making such a division was that the equations which are more strongly coupled are solved by the Newton-Raphson method. The equations which are not strongly coupled 
with the other set of equations are solved by the successive substitution method. Thus, the computer memory requirement can be significantly reduced while maintaining superior numerical convergence characteristics. To improve the convergence and stability of the numerical scheme, the successive substitution method is used to provide an initial guess for pressure and flow rate. Equations 2 and 3 are rewritten such that pressures and flow rates can be estimated at each node and branch.

In a typical unsteady calculation, the SAAS procedure consists of the following steps:

1. At the beginning of a new time step, provide the initial solution of all dependant variables in the flow domain e. g. pressure, resident mass, density and entropy at all internal and boundary nodes, flow rates at all branches.

2. Begin the outer iteration loop; the purpose of this loop is to calculate entropy and density at all internal nodes and flow resistance in the branches.

3. Solve mass conservation equation (Equation 2) in internal nodes, momentum conservation equation in branches (Equation 3) and equation of resident mass in internal nodes (Equation 6) by Newton Raphson method (Appendix - A ).

4. Solve entropy conservation equation by successive substitution method (Appendix B)

5. Calculate density and temperature from the equation of state for calculated pressure and entropy at each internal node. Viscosity is also computed from the thermophysical property correlation for calculated pressure and temperature.

6. Calculate flow resistance parameter $\left(\mathrm{K}_{\mathrm{f}}\right)$ of each branch. $\mathrm{K}_{\mathrm{f}}$ is a function of density and viscosity.

7. Calculate the maximum difference in values of entropy, density and flow resistance parameters between successive iterations. Steps 3 to 7 constitute one iteration cycle.

8. Repeat steps 3 to 7 until the maximum difference is less than the specified convergence criterion. Steps 2 to 8 constitute all operations required for one time step.

9. Repeat steps 1 to 8 until final time is reached.

\section{COMPUTER PROGRAM}

The numerical procedure described in the previous sections was incorporated into a general purpose computer program named Generalized ]lluid System Simulation Program (GFSSP) ${ }^{7}$. The computer program has three major parts. The first part consists of the subroutines for the preprocessor. The preprocessor allows the user to interactively create the flow network model consisting of nodes and branches. All of the input specifications, including the boundary conditions, are specified through the preprocessor. The second major part of the program consists of the subroutines that provide the initial conditions and then develop and solve all of the conservation equations in the flow network. The third part of the program consists of the thermodynamic property programs, GASP and WASP that provide the necessary thermodynamic and thermophysical property data required to solve the resulting system of equations. 
Figure 6 shows GFSSP's process flow diagram. The user runs the interactive preprocessor to generate the input data file. The input data file contains all the information necessary for the model. The solver module reads the input data file and produces the solution in conjunction with the thermodynamic property programs.

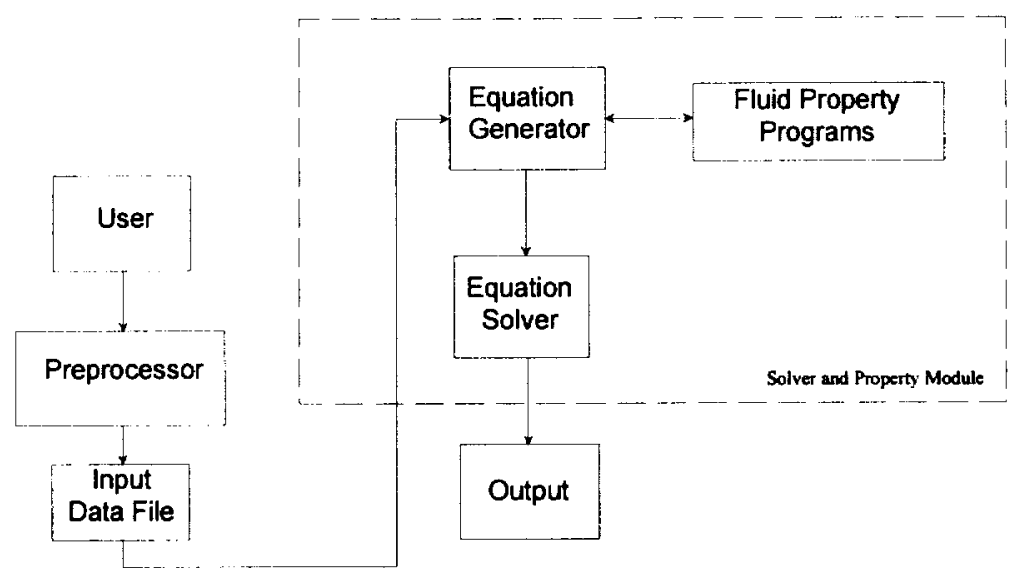

Figure 6 - GFSSP Process Flow Diagram

\section{RESULTS}

The feasibility, robustness and verification of the proposed method have been demonstrated by simulating five example problems. They are:

1. Flow system consisting of a pump, valve and pipe line.

2. Water distribution network.

3. Compressible flow through a converging-diverging nozzle.

4. Blow down of a pressurized tank.

5. Recirculating flow in a square cavity

Example 1 - Flow System Consisting of a Pump, Valve and a Pipe Line

A problem commonly encountered in fluid engineering is to match a pump's characteristics with the operating system's characteristics. The designer needs to know the flow rate in the system and the power consumed by the pump. The system considered for this example is shown in Figure 7.

The fluid system shown in Figure 7 was simulated with a finite volume model consisting of four nodes and three branches as shown in Figure 8. 


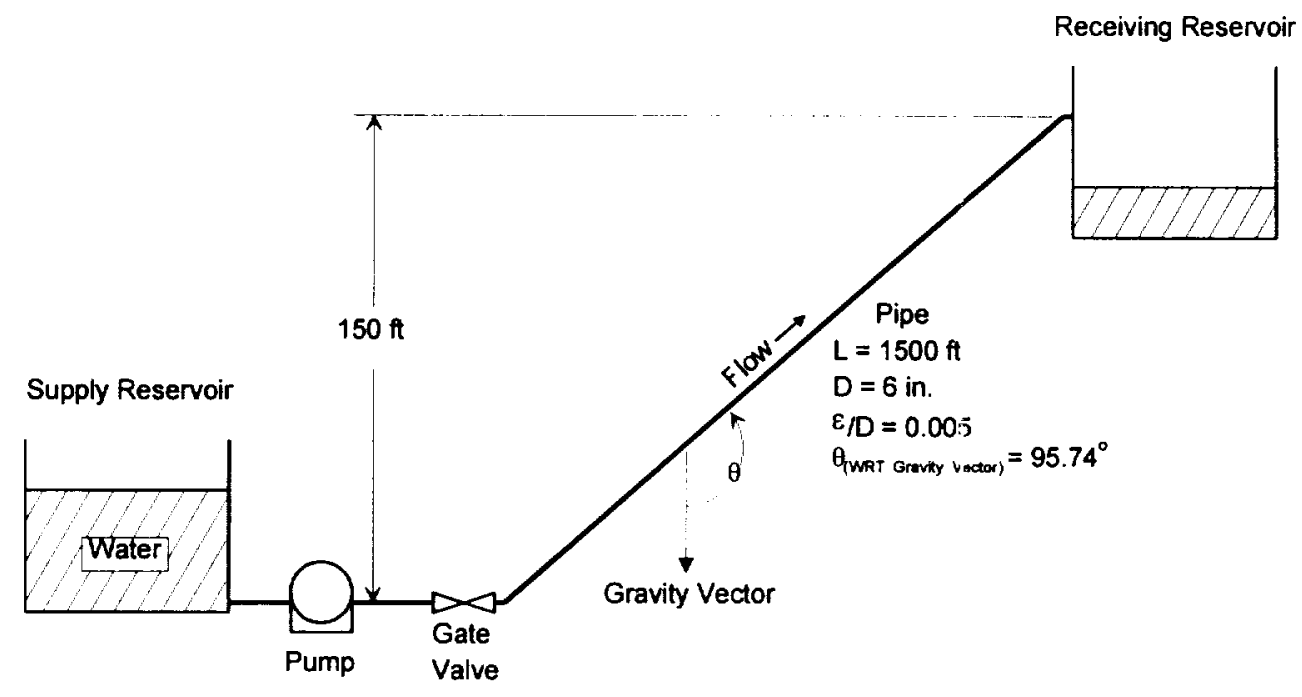

Figure 7 - Flow system consisting of a pump, valve and pipe line.

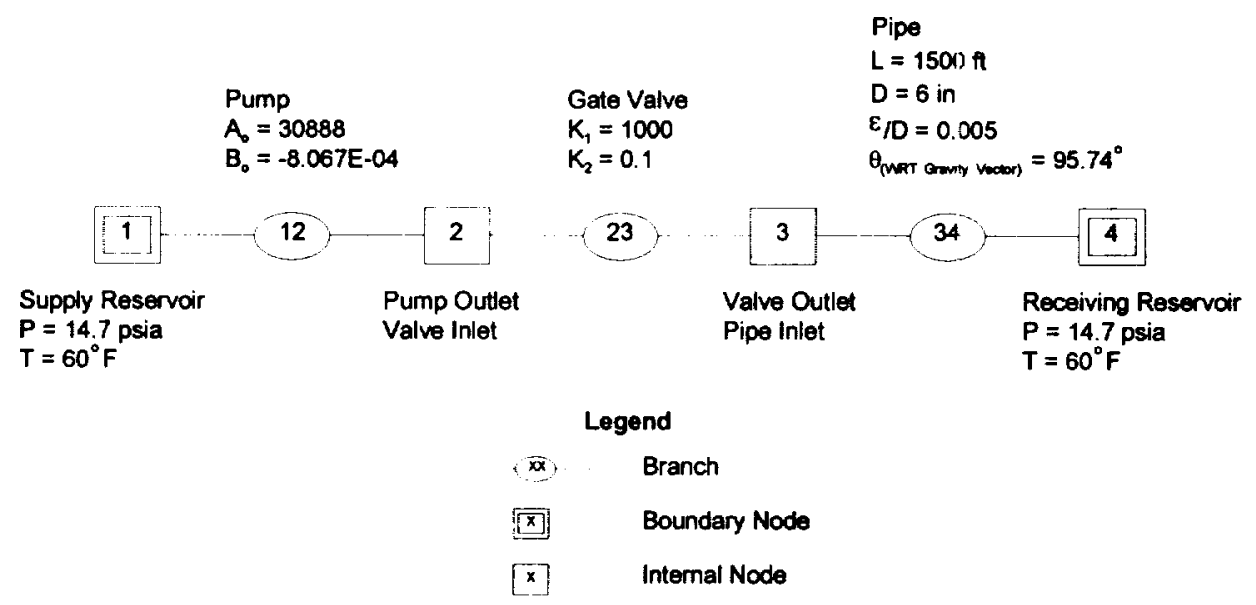

Figure 8 - Finite volume Model of Flow System Described in Example 1.

\section{Pump Model}

The pump was modeled as a momentum source in branch 12 . The pump characteristics were expressed as:

Where:

$$
\Delta \mathrm{p}=\mathrm{A}_{0}+\mathrm{B}_{0} \dot{\mathrm{m}}^{2}
$$

$$
\begin{aligned}
& \Delta p=\text { Pressure rise, } \mathrm{lbf} / \mathrm{ft}^{2} \\
& \dot{m}=\text { Flow rate, } \mathrm{lbm} / \mathrm{sec}
\end{aligned}
$$

The momentum source, $\mathrm{S}$, in Equation 3 was then expressed as: 
$\mathrm{S}=\Delta \mathrm{pA}$

Valve Model

The resistance in branch 23 representing the valve was computed by the two-K method of Hooper ${ }^{8}$. For this option, $K_{f}$ of Equation 3 was expressed as:

$$
\mathrm{K}_{\mathrm{f}}=\frac{\mathrm{K}_{1} / \mathrm{Re}+\mathrm{K}_{\infty}(1+1 / \mathrm{D})}{2 \mathrm{~g}_{\mathrm{c}} \rho_{\mathrm{u}} \mathrm{A}^{2}}
$$

Where:

$$
\begin{aligned}
& \mathrm{K}_{1}=\mathrm{K} \text { for the fitting at } \mathrm{Re}=1 ; \\
& \mathrm{K}_{\infty}=\mathrm{K} \text { for the fitting at } \mathrm{Re}=\infty \\
& \mathrm{D}=\text { Internal diameter of attached pipe, in. }
\end{aligned}
$$

The constants $K_{1}$ and $K_{\infty}$ for common fittings and valves are listed in reference 8 .

\section{Pipe Model}

The resistance in branch 34 was computed from the friction factor in the pipe line. The resistance coefficient, $\mathrm{K}_{\mathrm{f}}$, for a pipe with length, $L$, diameter, $D$, and surface roughness, $\varepsilon$ was expressed as:

$$
K_{f}=\frac{8 f L}{\rho_{u} \pi^{2}{ }^{5} g_{c}}
$$

Where $\rho_{u}$ is the density of the fluid at the upstream node of a given branch.

The Darcy friction factor, $f$, is determined from the Colebrook Equation ${ }^{9}$ which is expressed as:

$$
\frac{1}{\sqrt{f}}=-2 \log \left[\frac{\varepsilon}{3.7 D}+\frac{2.51}{\operatorname{Re} \sqrt{f}}\right]
$$

Where $\varepsilon / \mathrm{D}$ and Re are the surface roughness factor and Reynolds number respectively.

It took 28 iterations to satisfy the convergence criterion. The solutions are shown in Tables 2 and 3 . Table 2 shows pressures, temperatures, compressibility factors and density in nodes 2 and 3 . It may be noted that water was treated as a compressible fluid and the compressibility factor is very low as expected. 
Table 2. Predicted Solution of Example I at Internal Nodes

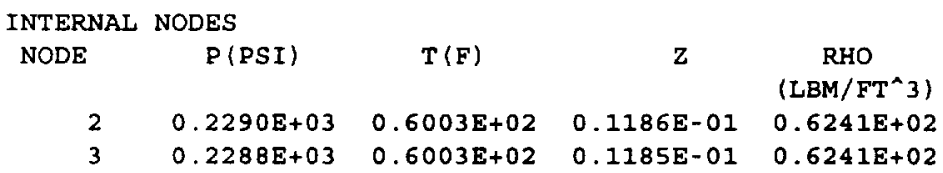

Table 3. Predicted Solution of Example 1 at Branches

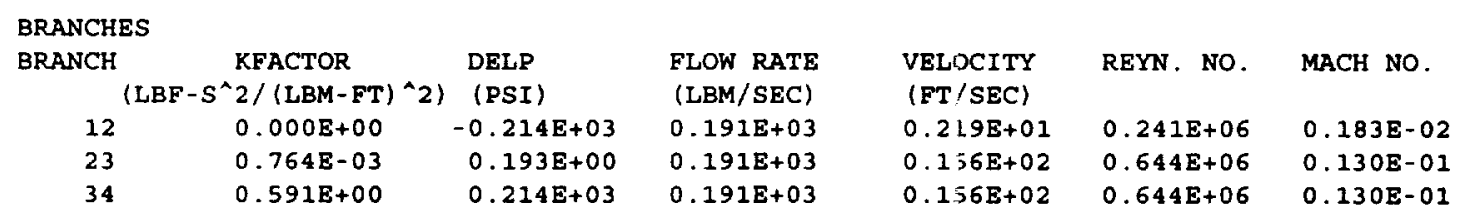

Table 2 shows $\mathrm{K}_{f}$, pressure drop, flow rate, velocity, Reynolds number and Mach number in each branch. The predicted flow rate is $191 \mathrm{lbm} / \mathrm{sec}$ and the pump is supplying 214 psid pressure rise to meet the system requirement. The predicted system and pump characteristics are shown in Figure 8 which also provides verification of the predicted operating point shown in Table 3.

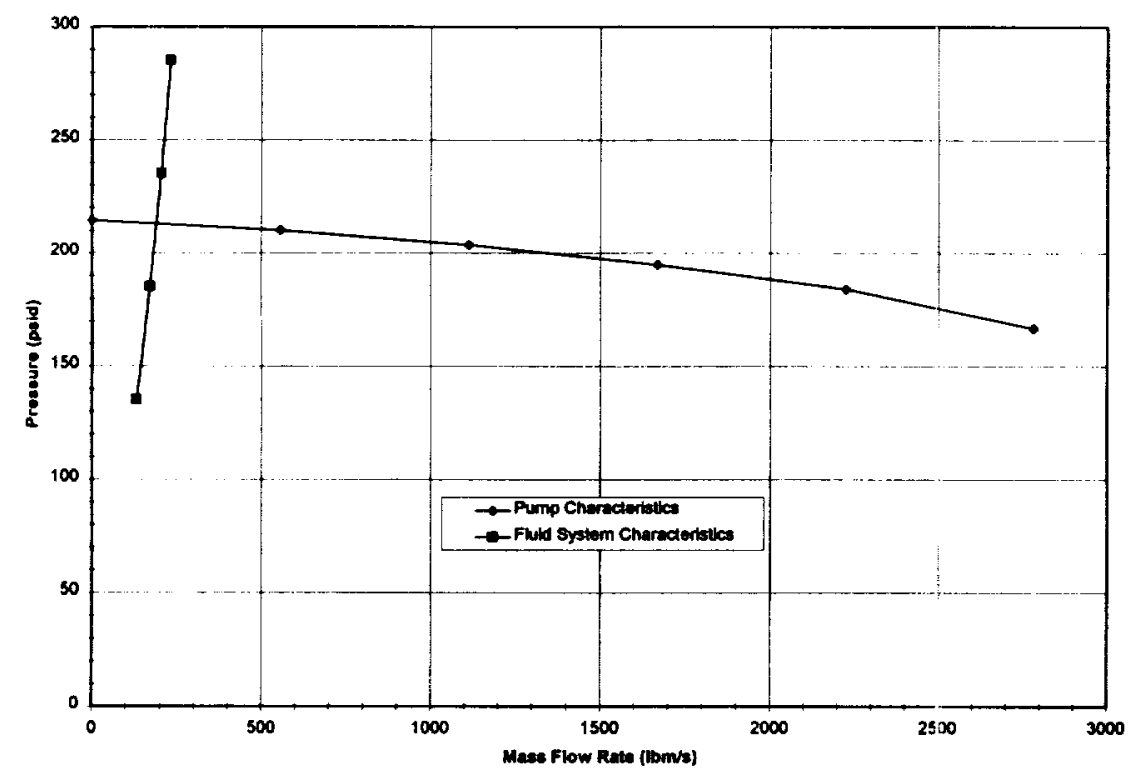

Figure 8 - Fluid System Operating Point

Example 2 - Water Distribution Network

In Example 1 we analyzed a single line pipe flow problem commonly encountered by pipeline designers. In this example, we consider an example associated with multipath systems which are commonly known as flow networks. In general, water supply systems are considered as flow networks, since nearly all such systems consist of many interconnecting pipes. A ten pipe (commercial steel) distribution system is shown in Figure 9. 


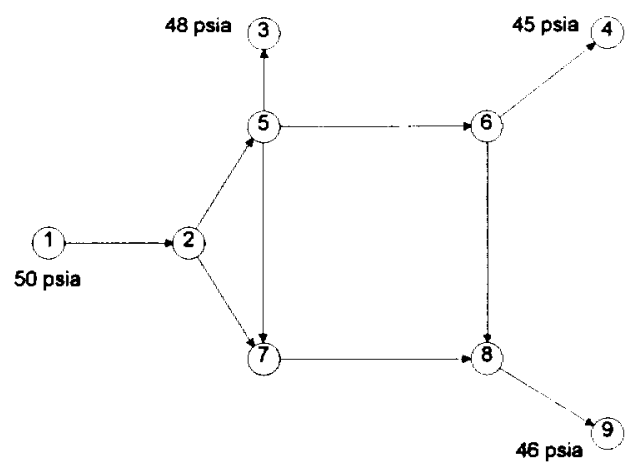

Figure 9 - A Schematic of Water Distribution Network

Table 4 - Water Distribution Network Branch Information

\begin{tabular}{|c|c|c|c|}
\hline Branch & Length(inches) & Diameter(inches) & $\begin{array}{c}\text { Roughness } \\
\text { Factor }\end{array}$ \\
\hline 12 & 120 & 6 & 0.0018 \\
\hline 25 & 2400 & 6 & 0.0018 \\
\hline 27 & 2400 & 5 & 0.0018 \\
\hline 57 & 1440 & 4 & 0.0018 \\
\hline 53 & 120 & 5 & 0.0018 \\
\hline 56 & 2400 & 4 & 0.0018 \\
\hline 64 & 120 & 4 & 0.0018 \\
\hline 68 & 1440 & 4 & 0.0018 \\
\hline 78 & 2400 & 4 & 0.0018 \\
\hline 89 & 120 & 5 & 0.0018 \\
\hline
\end{tabular}

The system shown in Figure 9 is modeled by using 9 nodes and 10 branches as shown in Figure 1. The fluid was assumed incompressible. Nodes $1,3,4$ and 9 are boundary nodes where the pressures are prescribed. Node 1 represents the inlet boundary node. Nodes 3 , 4 and 9 are outlet boundary nodes. All of the remaining nodes (2, 5, 6, 7 and 8) are internal nodes where the pressures are calculated. All of the branches in this circuit simulate pipes. The length, diameter and roughness factor of all branches are given in Table 4.

Figure 10 shows a comparison of flow rates between the present predictions and the Hardy Cross method. The comparison appears reasonable considering the fact that the Hardy Cross method assumes a constant friction factor in the branch while the present method computes the friction factor for each branch during every iteration. Therefore, as the flowrates change the friction factor also changes. 


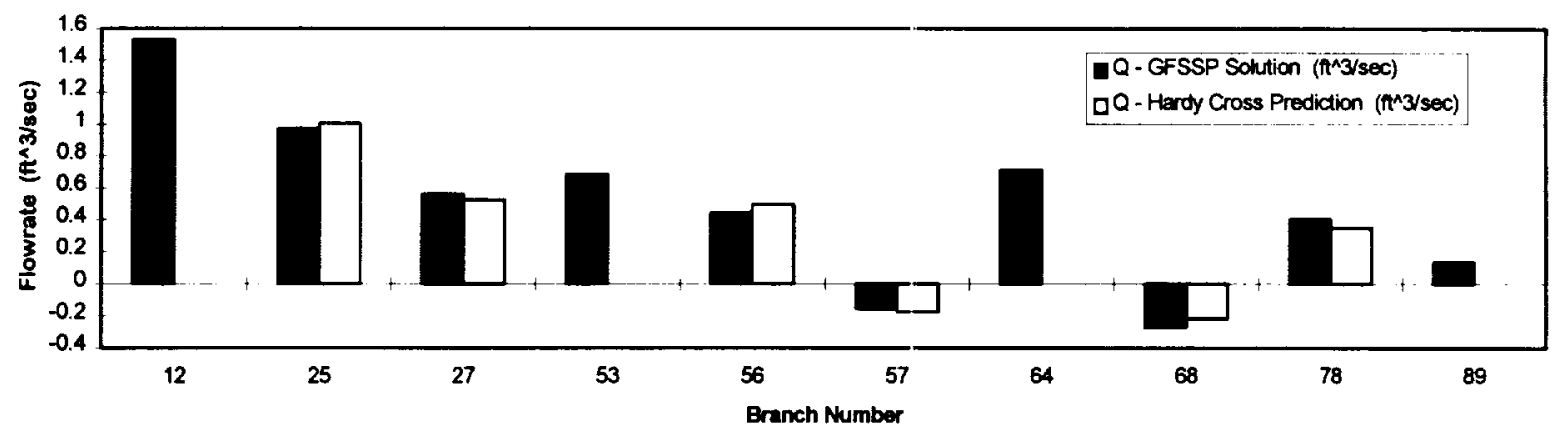

Figure 10 - A Flow Rate Comparison Between GFSSP and Hardy Cross Method Predictions

Example 3 - Compressible Flow in a Converging-Diverging Nozzle

In this example we will consider compressible flow in a converging-diverging nozzle to demonstrate the method's capability to handle compressibility. One of the characteristics of compressible flow in a duct is that the flow rate becomes independent of exit pressure after reaching a threshold flow rate. This threshold value is known as the choked flow rate and it is a function of inlet pressure and temperature. Flow in a confined duct becomes choked when the flow velocity equals the local velocity of sound. The purpose of this example is to investigate how accurately the present procedure can predict the choked flow rate in a converging-diverging nozzle.

The converging-diverging nozzle considered for this example is shown in Figure 11. The fluid considered was steam at $150 \mathrm{psia}$ and $1000^{\circ} \mathrm{F}$. The nozzle back pressure was varied from 134 psia to 15 psia.

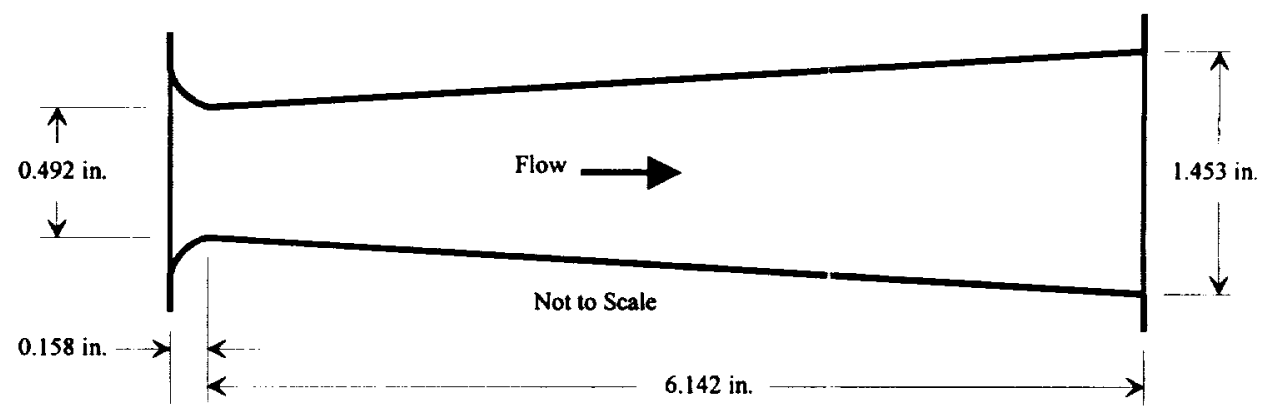

Figure 11 - Converging-Diverging Steam Nozzle Schematic

The fluid system shown in Figure 11 was simulated by seventeen nodes and sixteen branches. Nodes 1 and 17 are the boundary nodes representing the inlet and outlet of the nozzle. All of the remaining nodes are internal nodes connected in series. The outlet boundary node pressures were varied to include 134, 100,60,30 and 15 psia. Table 5 lists the model predicted mass flow rates with varying exit pressures. As expected, the mass flow rate increased as the exit pressure was decreased until the pressure ratio decreased 
below the critical pressure ratio. At this point and below, the mass flow rate remained constant due to choking of the flow at the nozzle throat.

Table 5 - Predicted Mass Flow Rate with Varying Exit Pressure

\begin{tabular}{|c|c|}
\hline $\begin{array}{c}\mathrm{P}_{\text {exit }} \\
\text { (psia) }\end{array}$ & $\dot{\mathrm{m}}(\mathrm{lbm} / \mathrm{s})$ \\
\hline 134 & 0.258 \\
\hline 100 & 0.301 \\
\hline 60 & 0.308 \\
\hline 30 & 0.308 \\
\hline 15 & 0.308 \\
\hline
\end{tabular}

The isentropic flow rate at the choked point was calculated to be $0.303 \mathrm{lbm} / \mathrm{sec}$ from the following relation:

$$
\dot{m}=\mathrm{A}_{\text {throat }} \mathrm{P}_{\text {inlet }} \sqrt{\frac{\mathrm{g}_{\mathrm{c}} \gamma}{\mathrm{R} \mathrm{T}_{\text {inlet }}}\left(\frac{2}{\gamma+1}\right)^{\left(\frac{\gamma+1}{\gamma-1}\right)}}
$$

Example 4 - Simulation of the Blow Down of a Pressurized Tank

In the previous examples we considered the simulation of steady state flow in a given flow circuit. In this example we will employ the capabilities of the unsteady flow formulation to simulate the process of blowing down a pressurized tank.

Consider a tank with an internal volume of $10 \mathrm{ft}^{3}$ containing nitrogen gas at a pressure and temperature of $100 \mathrm{psia}$ and $80^{\circ} \mathrm{F}$ respectively. The nitrogen is discharged into the atmosphere through an orifice with a 0.1 inch diameter until the pressure in the tank drops to 50 psia. The purpose of this example is to determine the blow down time and the pressure, mass flow rate and temperature history of the isentropic blow down process. These predicted values will then be compared with the analytical solution.
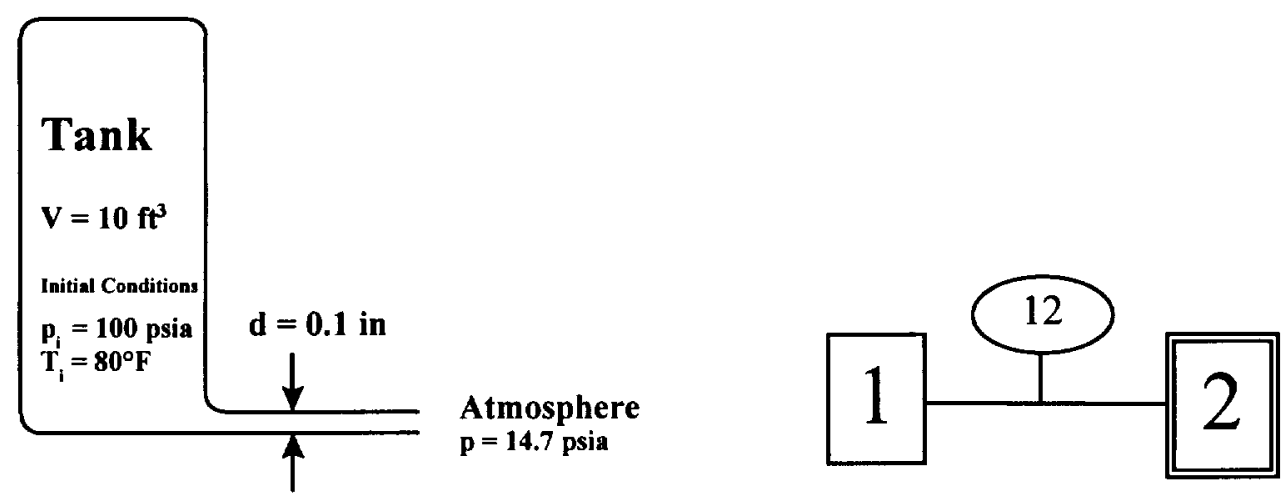

Figure 12 - Physical Schematic (a) and GFSSP Model (b) for Venting Nitrogen Tank 
The physical schematic for Example 8 is shown in Figure 12(a) and a schematic of the corresponding finite volume model is shown in Figure 12(b). The venting process can be modeled with two nodes and one branch. Node 1 is an internal node which represents the tank.

\section{Analytical Solution:}

The differential equation governing an isentropic blow down process can be written as:

$$
\left(\frac{p}{p_{i}}\right)^{(1-3 \gamma) / 2 \gamma} \frac{d\left(p / p_{i}\right)}{d \tau}=\frac{\gamma A}{\rho_{i} V} \sqrt{\gamma g_{c} p_{i} \rho_{i}}\left(\frac{2}{\gamma+1}\right)^{(\gamma+1) / 2(\gamma-1)}
$$

This is an initial value problem and the initial conditions are:

$$
\tau=0, \frac{p}{p_{i}}=1
$$

The analytical solution for $p / p_{i}$ is given by Moody ${ }^{10}$ as:

$$
\frac{p}{p_{i}}=\left[1+\left(\frac{\gamma-1}{2}\right)\left(\frac{2}{\gamma+1}\right)^{(\gamma+1) / 2(\gamma-1)} \sqrt{\frac{\gamma g_{c} p_{i}}{\rho_{i}}} \frac{A \tau}{V}\right]^{-2 \gamma /(\gamma-1)}
$$

The analytical and finite volume solutions obtained by GFSSP are compared in Figure 13. Excellent agreement was observed between the two solutions.

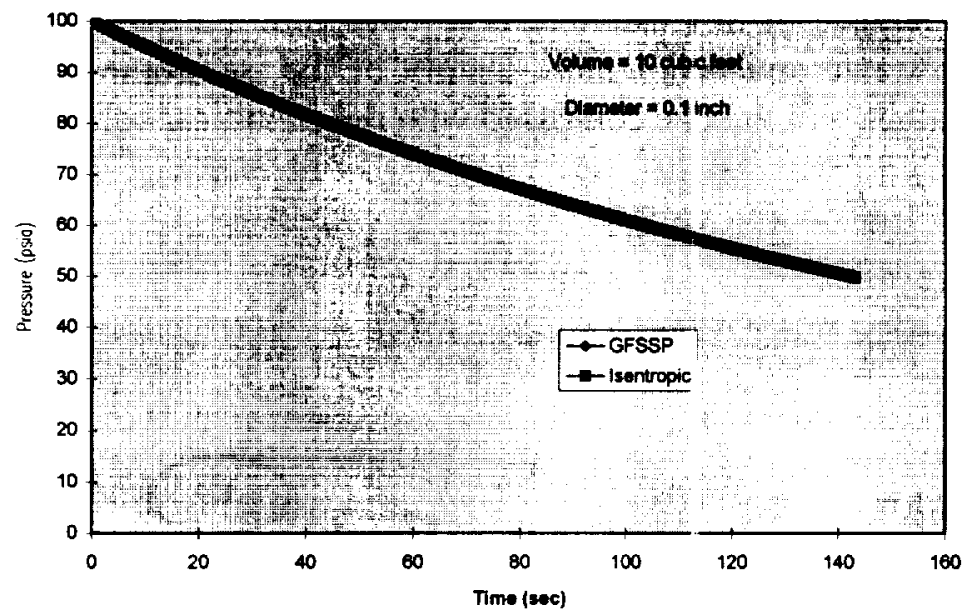

Figure 13 - Comparison of the Predicted Pressure History by Finite Volume Method using GFSSP and the Analytical Solution 


\section{Example 5 - Recirculating Flow in a Square Cavity}

Flow in a square cavity is induced by moving its top wall at a constant speed as shown in Figure 14. The density of the fluid is assumed constant at $1.00 \mathrm{lb}_{\mathrm{m}} / \mathrm{ft}^{3}$, and the viscosity of the fluid is assumed to be $1.00 \mathrm{lb}_{\mathrm{m}} /(\mathrm{ftsec})$. The bottom and side walls are fixed. The top wall is moving to the right at a constant speed of $100 \mathrm{ft} / \mathrm{sec}$. The corresponding Reynolds number for this situation is $\operatorname{Re}=100$.

Due to the complexity of the flow field, an analytical solution of this situation is not practical. Instead of an analytical solution, a well known numerical solution by Odus Burggraf ${ }^{11}$ was used as the benchmark solution. Burggraf used a $51 \times 51$ grid in his model of the square cavity.

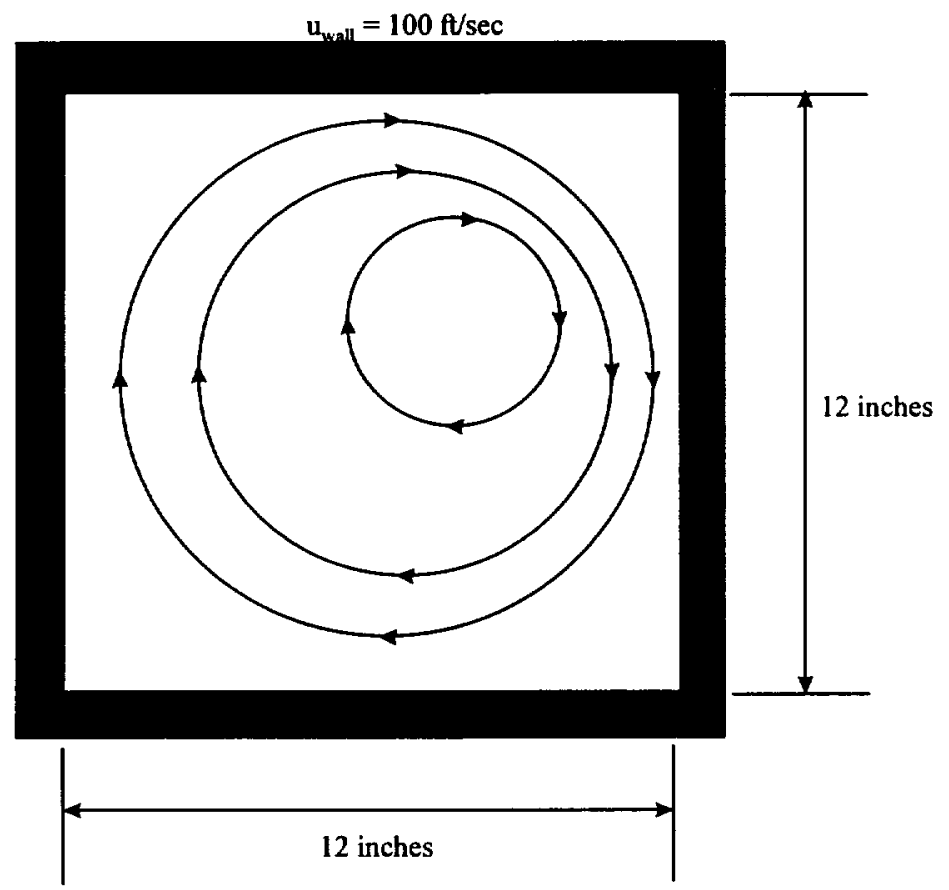

Figure 14 - Flow in a Shear Driven Square Cavity

The finite volume model of the driven cavity consists of 49 nodes (48 of which are internal) and 84 branches. For numerical stability, one node (Node 1) was assigned to be a boundary node with an arbitrary pressure of $100 \mathrm{psi}$. A unit depth (1 inch) was assumed for the required areas. The model is shown schematically in Figure 15. Modeling details are provided in GFSSP user's manual ${ }^{7}$ 


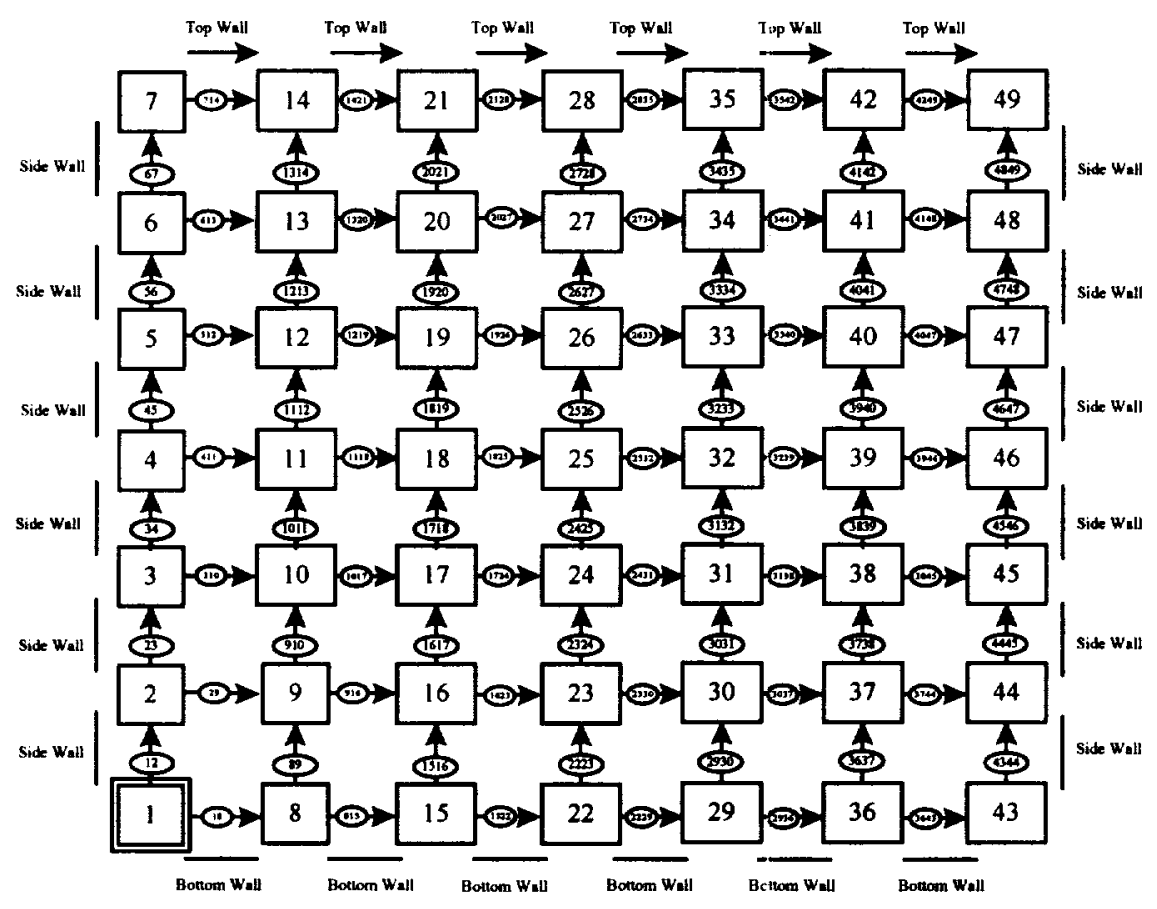

Figure 15 - Finite Volume Model of Flow in a Shear Driven Square Cavity

Figure 16 shows a comparison between the benchmark numerical solution and the finite volume predictions along a vertical plane at the horizontal midpoint. As can be seen in Figure 16, the results of this coarse grid model compare very favorably with the benchmark numerical solution of Burggraf.

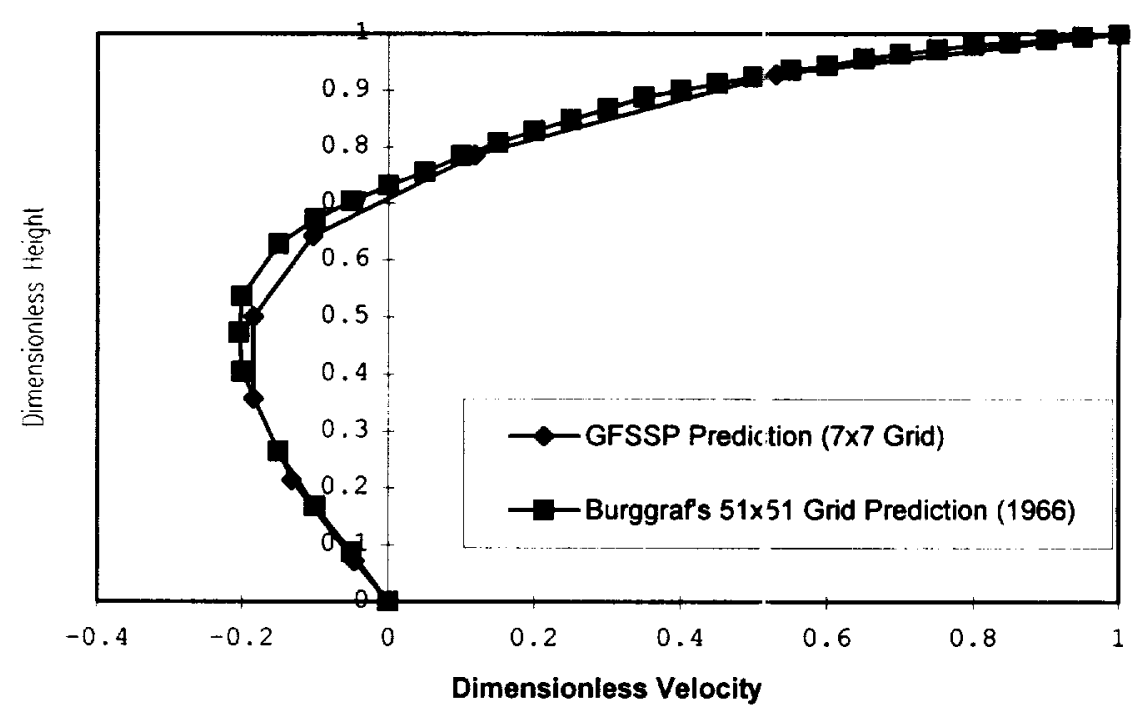

Figure 16 - Shear Driven Square Cavity Centerline Velocity Distribution 


\section{CONCLUSIONS}

1. The present paper has described a generally applicable numerical method to perform thermo-fluid dynamic analysis of a fluid system or component.

2. A novel unstructured co-ordinate system has been used; this allows the development of mass, momentum and entropy conservation equations in a complex flow network defined by interconnected nodes and branches.

3. The method described here uses a generalized momentum equation which considers an one-dimensional form for system level calculations. Fluid friction is calculated from the friction factor or loss coefficients. For component level analysis, the multidimensional form of the momentum equation is used. Fluid friction is, then, calculated from the local velocity gradient and viscosity. In addition, transverse transport of momentum is also computed.

4. Thermodynamics and fluid dynamics of the flow is linked through entropy and pressure. The second law formulation allows the calculation of entropy generation through heat and fluid flow. All thermodynamic and thermophysical properties are computed from local values of pressure and entropy.

5. The system of equations is solved by a novel numerical method which is a combination of successive substitution and Newton Raphson methods. The method has demonstrated a remarkable stability for both system and component level analysis.

6. The method has been incorporated into a general purpose computer program which is extensively being used in many aerospace engineering applications and the computer program, GFSSP, is available through COSMIC, NASA's Software Technology Transfer Center.

\section{ACKNOWLEDGEMENT}

This work was performed under Task No. 371-401 for Contract No. NAS8-40836 with Mr. Henry Stinson of Marshall Space Flight Center as Task Manager. The author would like to acknowledge the contribution of Mr. John W. Bailey, Paul Schallhorn and Todd Steadman of Sverdrup Technology in the development and testing of GFSSP. The author would also like to acknowledge the comments and suggestion of Mr. Tom Beasley of Sverdrup Technology during the course of this work.

\section{REFERENCES}

1. Hardy Cross, "Analysis of Flow in Networks of Conduits or Conductors", Univ. Ill. Bull. 286, November 1936.

2. Patankar, S. V. and Spalding, D. B., "A Calculation Procedure for Heat, Mass and Momentum Transfer in Three-Dimensional Parabolic Flows", Int. J. Heat Mass Transfer, Vol. 15, pp. 1787-1806, 1972.

3. Datta, A. B. and Majumdar, A. K., "Flow Distribution in Parallel and Reverse Flow Manifolds", Int. J. Heat \& Fluid Flow, Vol. 2, No. 4, pp. 253-262, 1980 
4. Bejan, A., Entropy Generation Through Heat and Fiuid Flow, New York, Wiley, 1982.

5. Bender, E., "Equations of State Exactly Representing the Phase Behavior of Pure Substances", Proceedings of the Fifth Symposium on Thermophysical Properties, ASME, pp. 227-235, 1970.

6. Hendricks, R. C., Baron, A. K., and Peller, I. C., "GASP - A Computer Code for Calculating the Thermodynamic and Transport Properties for Ten Fluids: Parahydrogen, Helium, Neon, Methane, Nitrogen, Carbon Monoxide, Oxygen, Fluorine, Argon, and Carbon Dioxide", NASA TN D)-7808, February, 1975.

7. Majumdar, A. K., Bailey, J. W., Schallhorn, P, Steadman, T., "A Generalized Fluid System Simulation Program to Model Flow Distribution in Fluid Networks", Sverdrup Technology Report No. 331-201-97-005, Contract No. NAS8-40836, October, 1997.

8. Hooper, W. B., "The Two-K Method Predicts Head Losses in Pipe Fittings.", Chem. Engr., Aug. 24, pp. 97-100, 1981.

9. Colebrook, C. F., "Turbulent Flow in Pipes, with Particular Reference to the Transition Between the Smooth and Rough Pipe Laws", J. Inst. Civil Engineering, London, vol. 11, pp. 133-156, 1938-1939.

10. Moody, F. J., "Introduction to Unsteady Thermofluid Mechanics", John Wiley, 1990.

11. Burggraf, O.R.: "Analytical and Numerical Studies of the Structure of Steady Separated Flows", Journal of Fluid Mechanics, Vol. 24, part 1, pp. 113-151, 1966.

\section{APPENDIX - A \\ Newton-Raphson Method of Solving Coupled Nonlinear System of Algebraic Equations}

The application of the Newton-Raphson Method involves the following 7 steps:

1. Develop the governing equations.

The equations are expressed in the following form:

$$
\begin{aligned}
& f_{1}\left(x_{1}, x_{2}, x_{3}, \ldots \ldots x_{n}\right)=0 \\
& f_{2}\left(x_{1}, x_{2}, x_{3}, \ldots \ldots x_{n}\right)=0 \\
& f_{n}\left(x_{1}, x_{2}, x_{3}, \ldots \ldots . x_{n}\right)=0
\end{aligned}
$$

If there are $\mathrm{n}$ number of unknown variables, there are $\mathrm{n}$ number of equations. 
2. Guess a solution for the equations.

Guess $x_{1}^{*}, x_{2}^{*}, x_{3}^{*}, \ldots \ldots x_{n}^{*}$ as an initial solution for the governing equations

3. Calculate the residuals of each equation.

When the guessed solutions are substituted into Equation A-1, the right hand side of the equation is not zero. The non-zero value is the residual.

$f_{1}\left(x_{1}^{*}, x_{2}^{*}, x_{3}^{*}, \ldots \ldots x_{n}^{*}\right)=R_{1}$

$f_{2}\left(x_{1}^{*}, x_{2}^{*}, x_{3}^{*}, \ldots \ldots x_{n}^{*}\right)=R_{2}$

$f_{n}\left(x_{1}^{*}, x_{2}^{*}, x_{3}^{*}, \ldots \ldots x_{n}^{*}\right)=R_{n}$

The intent of the solution scheme is to correct $x_{1}^{*}, x_{2}^{*}, x_{3}^{*}, \ldots \ldots x_{n}^{*}$ with a set of corrections $x_{1}^{\prime}, x_{2}^{\prime}, x_{3}^{\prime}, \ldots \ldots x_{n}^{\prime}$ such that $R_{1}, R_{2}, R_{3}, \ldots \ldots ., R_{n}$ are zero.

4. Develop a set of correction equations for all variables.

First construct the matrix of influence coefficients:

$\frac{\partial f_{1}}{\partial x_{1}} \frac{\partial f_{1}}{\partial x_{2}} \frac{\partial f_{1}}{\partial x_{3}} \ldots \ldots . . \frac{\partial f_{1}}{\partial x_{n}}$

$\frac{\partial f_{2}}{\partial x_{1}} \frac{\partial f_{2}}{\partial x_{2}} \frac{\partial f_{2}}{\partial x_{3}} \ldots \ldots . . \frac{\partial f_{2}}{\partial x_{n}}$

$\frac{\partial f_{n}}{\partial x_{1}} \frac{\partial f_{n}}{\partial x_{2}} \frac{\partial f_{n}}{\partial x_{3}} \ldots \ldots . . \frac{\partial f_{n}}{\partial x_{n}}$

Then construct the set of simultaneous equations for corrections:

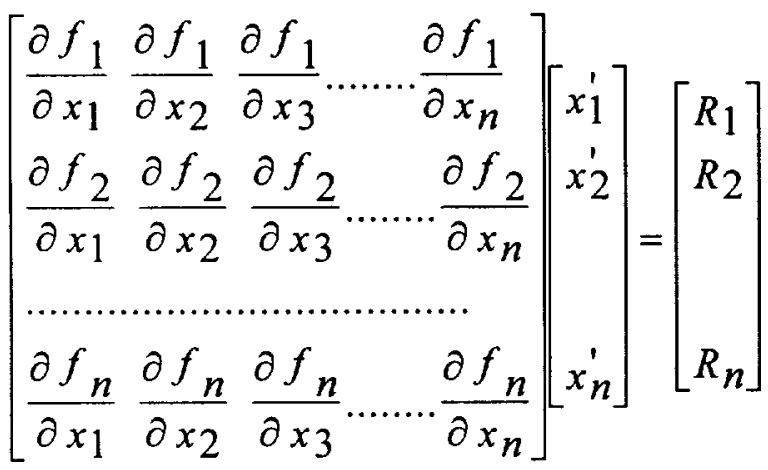


5. Solve for $x_{1}^{\prime}, x_{2}^{\prime}, x_{3}^{\prime}, \ldots \ldots x_{n}^{\prime}$ by solving the simultaneous equations.

6. Apply correction to each variable.

7. Iterate until the corrections become very small.

\section{APPENDIX - B}

\section{Successive Substitution Method of Solving Coupled Nonlinear System of Algebraic Equations}

The application of the successive substitution method involves the following steps:

1. Develop the governing equations:

$$
\begin{aligned}
& x_{1}=f_{1}\left(x_{1}, x_{2}, x_{3}, \ldots \ldots x_{n}\right) \\
& x_{2}=f_{2}\left(x_{1}, x_{2}, x_{3}, \ldots \ldots x_{n}\right)
\end{aligned}
$$

$$
x_{n}=f_{n}\left(x_{1}, x_{2}, x_{3}, \ldots \ldots x_{n}\right)
$$

If there are n number of unknown variables, there are n number of equations.

2. Guess a solution for the equations:

Guess $x_{1}^{*}, x_{2}^{*}, x_{3}^{*}, \ldots \ldots x_{n}^{*}$ as an initial solution for the governing equations.

3. Compute new values of $x_{1}, x_{2}, x_{3}, \ldots \ldots x_{n}$ by subsituting $x_{1}^{*}, x_{2}^{*}, x_{3}^{*}, \ldots \ldots x_{n}^{*}$ in the right hand side of Equation $B-1$.

4. Under-relax the computed new value:

$x=(1-\alpha) x^{*}+\alpha x$

where $\alpha$ is the under-relaxation parameter.

5. Replace $x_{1}^{*}, x_{2}^{*}, x_{3}^{*}, \ldots \ldots x_{n}^{*}$ with the computed value of $x_{1}, x_{2}, x_{3}, \ldots \ldots x_{n}$ from Step 4.

6. Repeat Steps 3 to 5 until convergence. 